\title{
Assessing the Cervical Range of Motion in Infants With Positional Plagiocephaly
}

\author{
Massimiliano Murgia, PhD, MD, ${ }^{*}$ Teresa Venditto, MD, ${ }^{\dagger}$ Marco Paoloni, MD, PhD, ${ }^{\dagger}$ \\ Brunilda Hodo, MD, ${ }^{\dagger}$ Rosaria Alcuri, MD, Andrea Bernetti, MD, ${ }^{\dagger}$ Valter Santilli, MD, ${ }^{\dagger \dagger}$ \\ and Massimiliano Mangone, $P h D^{\ddagger}$
}

\begin{abstract}
Purpose: To determine if infants with positional plagiocephaly have limitations of active and passive cervical range of motion measured with simple and reliable methods.

Methods: The examiners assessed bilateral active and passive cervical rotations and passive cervical lateral flexion. Cervical assessment was performed twice by 2 different physicians to assess intertester reliability. To assess intratester reliability the first investigator performed a second examination 48 hours after the first one.

Results: One-hundred nine subjects were analyzed; $70.7 \%$ of the sample had head positional preference on the right, while $29.3 \%$ had head positional preference on the left $\left(\chi^{2} 35.52, P<0.001\right)$. Cervical rotations and lateral flexion showed reliable levels of agreement for intra and intertester reliability.

Conclusions: The most limited range of motion in infants with positional plagiocephaly was cervical active rotation which affected more than $90 \%$ of patients. Passive cervical rotations and lateral flexion were limited in more than $60 \%$ of patients.
\end{abstract}

Key Words: Cervical assessment, cervical range of motion, positional plagiocephaly

(J Craniofac Surg 2016;27: 1060-1064)

$P$ ositional or deformational plagiocephaly (PP) is the most common infant cranial asymmetry characterized by an asymmetrical flattening of the skull and it is possibly associated with an increased risk of developmental delays in toddlers. ${ }^{1}$ Its incidence has significantly increased over the past few decades ${ }^{2}$ following the recommendation of the American Academy of Pediatrics to place infants in a supine sleeping position to avoid Sudden Infant Death Syndrome. ${ }^{3}$ Particularly, the incidence in infants at 7 to 12 weeks of age was estimated to be $46.6 \%{ }^{4}$ and the prevalence varies between $6.1 \%$ and $13.0 \%$ at birth, $16.0 \%$ and $22.1 \%$ at age 6 to 7 weeks,

From the * Physical Medicine and Rehabilitation Unit, Azienda Policlinico Umberto I; †Board of Physical Medicine and Rehabilitation, Department of Anatomy, Histology, Forensic Medicine and Orthopedics, "Sapienza" University, Rome; and †Don Carlo Gnocchi ONLUS Foundation, Milan, Italy.

Received August 19, 2015.

Accepted for publication March 5, 2016.

Address correspondence and reprint requests to Teresa Venditto, MD,

Department of Physical Medicine and Rehabilitation. Board of Physical

Medicine and Rehabilitation, Department of Anatomy, Histology,

Forensic Medicine and Orthopedics. "Sapienza" University, Piazzale

Aldo Moro, 500185 Rome, Italy; E-mail: teresa.venditto@hotmail.it

The authors report no conflicts of interest.

Copyright $(12016$ by Mutaz B. Habal, MD

ISSN: $1049-2275$

DOI: $10.1097 /$ SCS.0000000000002644
$19.7 \%$ at age 4 months, $9.2 \%$ at age 8 months, and $6.8 \%$ at age 12 months. $^{5}$

The risk of PP is increased for infants who required assisted deliveries, are first-born children, are male, have high cumulative exposure to supine positioning, and have neck problems such as infant difficulty turning head, decreased cervical rotation, limited passive cervical rotation, and limited active cervical rotation. The pathophysiologic mechanisms underlying this condition are poorly understood. $^{6-8}$

Abnormal prenatal and/or postnatal external forces acting on the malleable and growing cranium in the first months of life may be a cause unilateral occipital flattening and, in severe patients, even misalignment of the ears on the axial plane and asymmetry of the face. A strong association between congenital muscular torticollis (CMT), positional preference, and the onset of the PP has been suggested. ${ }^{9}$ Congenital muscular torticollis is a congenital musculoskeletal disorder characterized by unilateral shortening of the sternocleidomastoid muscle usually presenting during infancy. ${ }^{10}$ The presence of muscular damage or an imbalance in the neck after birth might, in fact, determine a "position of comfort" for the head, which could result in persistent rotation, thereby causing a flatten of the head. Alternatively, the presence of PP at birth could induce the development of a postnatal torticollis. ${ }^{11}$ To date, uncertainty still exists about the prevalence of cervical range of motion (ROM) limitations in infants with PP.

Measuring cervical ROM in patients with PP should be an essential part of the physical examination to identify factors, such as head rotation preferences to 1 side or decreased active cervical ROM, which might contribute to the development of PP. ${ }^{12}$ It is also essential to assess the severity of $\mathrm{PP}$, to provide appropriate nonsurgical or surgical therapeutic interventions as well as to verify their effectiveness.

We hypothesize that a high percentage of infants with PP might have both active and passive cervical ROM restrictions, and that these limitations could be detected by simple and reliable maneuvers during a standard physical examination.

The objective of the present research was, therefore, to determine if infants diagnosed with PP have limitations of active and passive cervical range of motion measured with simple and reliable methods.

\section{METHODS}

This study was designed as an uncontrolled, prospective patient series. From January 2011 to June 2013, all infants who referred to our Physical Medicine and Rehabilitation outpatient service for the assessment of cranial asymmetry were screened for eligibility.

Patients of both sex aged between 0 and 18 months with a diagnosis of PP were included in the present study. We excluded infants with a diagnosis of synostotic plagiocephaly, CMT, and suspected dysmorphism of the spine. ${ }^{13}$

All parents agreed to participate in the study and signed an informed consent form in accordance with the Declaration of Helsinki. 
Medical history of patients was obtained from parents and a standard physical examination was performed on each patient before the cervical ROM measurements were taken. The degree of skull asymmetry was evaluated according to Argenta classification. ${ }^{14,15}$ It distinguishes 5 types of PP, based on the severity of the asymmetry of the skull, ear position and face as follows: restricted to the back of the skull, adds malposition of the affected ear, adds forehead deformity, adds facial deformity, and adds abnormal vertical growth. ${ }^{14}$

The assessment of active and passive cervical rotations and passive lateral flexion was performed twice on every patient by 2 different physicians (examiner 1 and examiner 2), to assess intertester reliability. The assessors performed their evaluation without being made aware of one another and another's assessment 1 to 24 hours apart.

Furthermore, examiner 1 performed a second examination 48 hours after the first one to assess intratester reliability.

Those who participated in the study did not receive any form of treatment between the first and second examinations.

The infant was placed supine on an examination table with his shoulders in a stabilized position to observe the spontaneous attitude of the head. The assessment of bilateral active and passive cervical rotations was performed taking as reference the baby's chin and the shoulder ipsilateral to the rotation.

The infant was stimulated to perform an active rotation of the head toward both sides using a colored object and a sound or the maternal voice and face. Once the infant reached the maximum degree of active rotation, the physician softly forced the head rotation to obtain the full passive ROM. Moreover, the presence of stress signs in baby's facial expression, crying, and marked opposition to the forced rotation were considered to determine the end of rotation ROM. ${ }^{16}$

Both active and passive cervical rotation ROM were categorized as follows (Fig. 1): full ROM, chin goes beyond the ipsilateral shoulder; mild limitation, chin reaches the ipsilateral shoulder without going beyond it; moderate limitation, chin goes beyond half clavicle but does not reach the should and severe limitation, chin does not go beyond half clavicle.

To assess the passive cervical lateral flexion, the infant's ear was approached to the ipsilateral shoulder, by placing examiner's hand at the level of the occipital bone of the child, with the contralateral shoulder held stable by the examiner. Cervical lateral flexion was

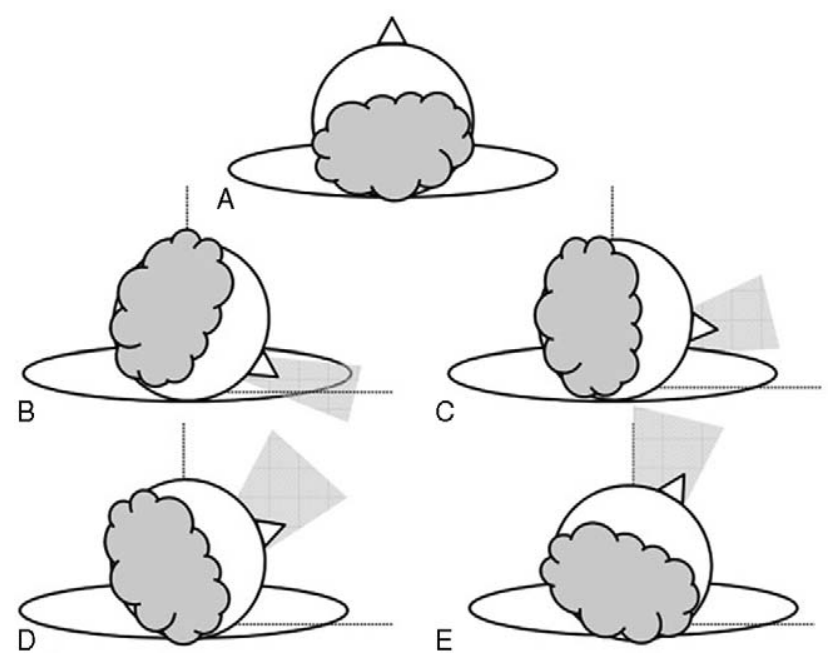

FIGURE 1. Active and passive cervical rotation ROM. (A) Neutral position. (B) Full ROM. (C) Mild limitation. (D) Moderate limitation. (E) Severe limitation. ROM, range of motion.

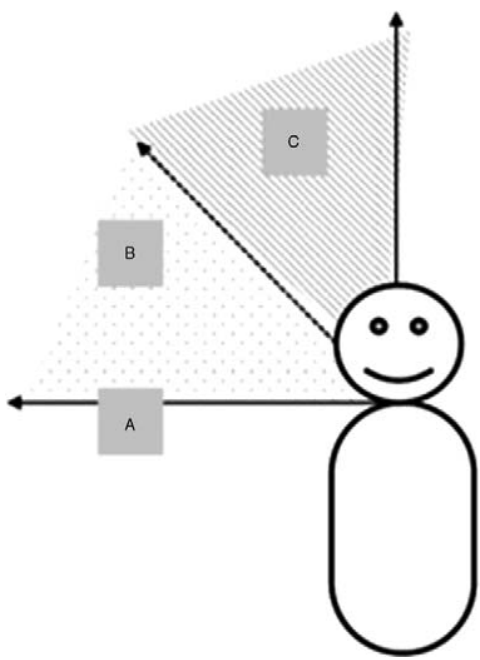

FIGURE 2. Passive cervical lateral flexion. (A) Full range of motion. (B) Moderate limitation. (C) Severe limitation.

categorized as follows (Fig. 2): full ROM, the ear reaches the shoulder; moderate limitation, the ear goes beyond $45^{\circ}$ of inclination but does not reach the shoulder; severe limitation, the ear does not go beyond $45^{\circ}$ of inclination.

The starting and ending positions of the cervical spine were noted for each measurement maintaining the cervical neutral throughout the measurements.

Each motion, passive and active cervical rotation and cervical lateral flexion, was repeated 3 times, and the best measurements (ie, that with the greatest ROM achieved) were thus analyzed.

\section{Statistical Analysis}

All the data were analyzed by an individual researcher. After verifying the normal distribution of the analyzed measurements using a Kolmogorov-Smirnov test to verify that the variables were normally distributed, parametric tests were used.

Cohen Kappa was used for the assessment of both intra and intertester reliability of categorical data. Cohen Kappa takes on values between -1 and 1 , with 1 indicating perfect agreement between the testers, -1 indicating perfect disagreement, and 0 indicating that any agreement is completely due to chance. It has been suggested that Kappa values above $80 \%$ demonstrate excellent agreement, values from $60 \%$ to $80 \%$ substantial levels of agreement, values from $40 \%$ to $60 \%$ moderate agreement, and values below $40 \%$ poor to fair agreement. ${ }^{17}$

The $\chi^{2}$ test was used to analyze the prevalence of right or left head positional preference and the side prevalence of the active and passive cervical rotation and the passive lateral flexion. The severity of ROM limitations was assessed by means of $\chi^{2}$ test.

Correlation analysis was performed to investigate the relationships of Argenta classification with respect to age, sex, and cervical ROM limitations. Correlation analysis was also performed between the degree of cervical imbalance and age at examination.

Correlation coefficients were interpreted as follows: 0.00 to 0.19 : very weak correlation; 0.20 to 0.39 : weak correlation; 0.40 to 0.69: moderate correlation; 0.70 to 0.89 : strong correlation; and 0.90 to 1 : very strong correlation. ${ }^{18}$

Statistical Package for Social Sciences (SPSS) version 18 was used for calculations. Differences with $P$ values $\leq 0.05$ were considered to be statistically significant, and all results were expressed with a $95 \%$ confidence interval. 


\begin{tabular}{ll}
\hline TABLE 1. Baseline Characteristics of Patients \\
\hline Number of patients & 109 \\
Age (mo) & $4.6 \pm 2.1 \mathrm{SD}$ (range: $1.5-13)$ \\
Sex & $70 \mathrm{M}, 39 \mathrm{~F}$ \\
Head positional preference & $77 \mathrm{R}, 32 \mathrm{~L}$ \\
Position assumed during sleep & $77 \mathrm{R}, 32 \mathrm{~L}$ \\
& 21 asymmetry on occipital side \\
& 31 malposition of ipsilateral ear \\
Argenta classification & 30 ipsilateral frontal bone protrusion \\
& 22 asymmetry ipsilateral facial \\
& 5 temporal bulging or abnormal vertical growth \\
& $14 \%(\mathrm{~N}=15)$ premature \\
& $32 \%(\mathrm{~N}=34)$ first gravidity \\
& $5.5 \%(\mathrm{~N}=6)$ breech presentation \\
Risk Factors & $46 \%(\mathrm{~N}=50)$ assisted delivery \\
& $7.3 \%(\mathrm{~N}=8)$ Oligoidramnios
\end{tabular}

F, female; L, left; M, male; R, right; SD, standard deviation.

\section{Sample Size}

Power calculations were based on a correlation value hypothesis $\mathrm{H} 1$ of -0.46 . Assuming a Cohen Kappa coefficient value of 0.6 , a $2-$ tailed $\alpha$ value of 0.05 (sensitivity $95 \%$ ) and a $\beta$ value of 0.05 (study power: $95 \%$ ), we determined that at least 70 subjects were required (G Power3 power analysis program).

\section{RESULTS}

One-hundred nine subjects (70 males and 39 females), with a mean age of 4.6 months (standard deviation [SD]: 2.1 months; range [R]: $1.5-13$ months) met the inclusion criteria. The baseline characteristics of the sample are reported in Table 1.

Kappa statistics and 95\% CIs for the cervical active and passive ROM are presented in Table 2. Intertester reliability was 0.80 for active cervical rotation, 0.83 for passive cervical rotation, and 0.49 for passive lateral flexion, indicating excellent and moderate levels of agreement. Intratester reliability was 0.72 for active cervical rotation, 0.73 for passive cervical rotation, and 0.41 for passive lateral flexion, indicating excellent and moderate levels of agreement.

As far as the direction of detected ROM limitations, in all patients the active and passive cervical rotations were limited contralateral, whereas passive cervical lateral flexion was limited ipsilateral to the side of head positional preference.

The $70.7 \%(\mathrm{~N}=77)$ of sample had head positional preference on the right, while the $29.3 \%(\mathrm{~N}=32)$ had head positional preference on the left $\left(\chi^{2} 35.52, P<0.001\right)$ (Table 3$)$. Among patients with head positional preference on the right, the $93 \%(\mathrm{~N}=71)$ had a limitation of active cervical rotation on the left $\left(\chi^{2} 120.42\right.$, $P<0.001)$, the $60 \%(\mathrm{~N}=46)$ had a limitation of passive cervical rotation on the left side $\left(\chi^{2} 77.69, P<0.001\right)$, the $62 \%(\mathrm{n}=48)$ had a significant limitation of passive lateral flexion on the right side $\left(\chi^{2}=77.69, P<0.001\right)$. Among patients with head positional preference on the left, the $94 \%(\mathrm{~N}=30)$ had a limitation of active cervical rotation on the right side $\left(\chi^{2} 120.42, P<0.001\right)$; the $75 \%$ $(\mathrm{N}=24)$ had a limitation of passive cervical rotation on the right side $\left(\chi^{2} 35.26, P<0.001\right)$, the $62.5 \%(\mathrm{~N}=20)$ had a limitation of passive tilt on the left side $\left(\chi^{2} 26.26, P<0.001\right)$.

Correlation analysis showed a not statistically significant relationship between cranial asymmetry and age $(r=0.102$, $P=0.40)$ and sex $(r=0.14, P=0.22)$. We also found a not statistically significant relationship between the degree of skull asymmetry and active cervical rotation $(r=0.107, P=0.268)$, a significant relationship between degree of skull asymmetry and passive cervical rotation $(r=0.369, P=0.000)$, and a significant relationship between degree of skull asymmetry lateral flexion $(r=0.319, P=0.000)$.

Correlation analysis showed a not statistically significant relationship between the degree of cervical imbalance and age at examination (Table 4).

\section{DISCUSSION}

The main results of the present study were that in patients with PP, active and passive cervical ROM limitations are common, being detectable in up to $93 \%$ of subjects. As far as we know, this is the first study to report such a high prevalence of cervical ROM limitation in PP. The most limited ROM was cervical active rotation which was decreased in $>90 \%$ of patients. Moreover, passive rotations and lateral flexion were limited in more than $60 \%$ of patients.

Furthermore, the direction of detected ROM limitations was in all patients contra laterally to the side of head positional preference for the rotations, and ipsilateral to the side of head positional preference for lateral flexion. The cervical spine is surrounded by a complex arrangement of muscles that contribute to static and dynamic control of the head and neck. Probably, the head positional preference causes a different morphological evolution between the muscle layers that encapsulate the spine producing a variation in their mechanical effect on the spine.

As far as factors influencing the magnitude of cranial asymmetry, we found a significant correlation between the degree of skull asymmetry and both passive cervical rotation and lateral flexion, but not with active cervical rotation. Age and sex do not appear to influence the cranial asymmetry.

Moreover, although the presence or absence of congenital or acquired torticollis is an important factor that affects gross motor development in infants with plagiocephaly, ${ }^{19}$ we found not significant relationship between the degree of cervical imbalance and age at examination. Therefore, on the basis of our data, we cannot definitively determine if this muscular imbalance might represent the cause or the effect of PP; however, we can confirm the data from the previous literature regarding the strong association between these 2 conditions. ${ }^{5}$

Future studies are needed to determine if early evaluations of cervical ROM limitations and physiotherapy, along with tummy time and general positioning rules, could lead to a reduction in the rate of progression of PP over time, as well as to a reduction in the use of cranial orthoses or surgery.

TABLE 2. Intra and Tester-Operator Reliability Coefficients for Cervical Range of Motion Assessment

\begin{tabular}{|c|c|c|c|c|}
\hline & Intratester Agreement (Kappa) & SE of Kappa & Intertester Agreement (Kappa) & SE of Kappa \\
\hline Active cervical rotation & $0.72(0.51-0.93)$ & 0.10 & $0.80(0.59-1)$ & 0.09 \\
\hline Passive cervical rotation & $0.73(0.49-0.97)$ & 0.13 & $0.83(0.66-1)$ & 0.08 \\
\hline Passive lateral flexion & $0.41(0.35-0.77)$ & 0.18 & $0.49(0.11-0.77)$ & 0.07 \\
\hline
\end{tabular}

Mean values of Kappa statistics (95\% CI) and standard errors (SE) are represented for each tested movement. 


\begin{tabular}{|c|c|c|c|c|c|c|}
\hline \multirow[b]{2}{*}{ Cervical ROM } & \multicolumn{3}{|c|}{$\begin{array}{l}\text { Right Head Positional } \\
\text { Preference }(N=77)\end{array}$} & \multicolumn{3}{|c|}{$\begin{array}{l}\text { Left Head Positional } \\
\text { Preference }(\mathbf{N}=32)\end{array}$} \\
\hline & Side & $\chi^{2}$ & $P$ Value & Side & $\chi^{2}$ & $P$ Value \\
\hline Active rotation & Left & 120.42 & $<0.001$ & Right & & 0.001 \\
\hline Mild (N) & 44 & 43.14 & 0.001 & 16 & 15.60 & 0.001 \\
\hline Moderate $(\mathrm{N})$ & 19 & & & 12 & & \\
\hline Severe $(\mathrm{N})$ & 8 & & & 2 & & \\
\hline Passive rotation & Left & 77.69 & $<0.001$ & Right & & 0.001 \\
\hline Mild (N) & 33 & 15.69 & 0.001 & 21 & 47.62 & 0.001 \\
\hline Moderate $(\mathrm{N})$ & 13 & & & 1 & & \\
\hline Severe $(\mathrm{N})$ & - & & & 1 & & \\
\hline Pasive lateral flexion & Right & 77.69 & $<0.001$ & Left & & 0.001 \\
\hline Mild (N) & 39 & 35.04 & 0.001 & 16 & 12.10 & 0.001 \\
\hline Moderate $(\mathrm{N})$ & 9 & & & 4 & & \\
\hline Severe $(\mathrm{N})$ & - & & & - & & \\
\hline
\end{tabular}

$\mathrm{N}$, number.

Our data contrasts with a previous study conducted by Rogers et $\mathrm{al}^{20}$ which measured cranial asymmetry using an anthropometric spreading caliper. This study found statistically significant negative correlation between age and head rotational asymmetry $(r=-0.200 ; P=0.004)$ and a significant positive correlation between cranial asymmetry and head rotational asymmetry ( $r=0.142 ; P=0.043$ ). However, according to the interpretation of the Pearson correlation coefficient, ${ }^{18}$ they showed weak and very weak correlations.

We considered the cervical ROM examination with simple maneuvers that classified the neck limitations as mild, moderate, and severe, in terms of anatomical landmarks as described above.

Health care professionals use a variety of methods to evaluate cervical spine ROM, but in order for a measure of ROM to be clinically useful, it must be first reliable; this refers to the consistency of a measurement across time, patients, or observers. ${ }^{21}$ In our study the active and passive cervical rotations demonstrated the highest level of intra and intertester reliability. In particular, for the active and passive cervical rotations, substantial and excellent levels of agreement were found for intra and intertester reliability, respectively. Moreover, for passive side bending moderate agreement was found for both intra and intertester reliability.

Cervical passive ROM rotation is considered to be normal at $110^{\circ}$, while in lateral flexion the variation is $40^{\circ}$ to $90^{\circ}$ in children with congenital muscular torticollis. ${ }^{22,23}$ However, the references of ROM values in healthy infants are insufficient. Indeed, only Ohman and Beckung ${ }^{24}$ evaluated in 38 healthy infants the cervical passive

TABLE 4. Correlation Between the Degree of Cervical Imbalance and Age of Subjects

\begin{tabular}{|c|c|c|c|c|c|}
\hline & \multicolumn{2}{|c|}{$\begin{array}{c}\text { Right Head } \\
\text { Positional Pre- } \\
\text { ference }\end{array}$} & \multicolumn{3}{|c|}{ Left Head Positional Preference } \\
\hline & Age & $P$ Value & & Age & $P$ Value \\
\hline Left active rotation & -0.15 & 0.12 & Right active rotation & -0.26 & 0.07 \\
\hline Left passive rotation & -0.11 & 0.27 & Right passive rotation & -0.31 & 0.03 \\
\hline Right lateral flexion & 0.07 & 0.43 & Left lateral flexion & -0.15 & 0.28 \\
\hline
\end{tabular}

rotation and lateral flexion, using an arthrodial protractor. The infants were laying supine on the examination table with the shoulders held stable and the examiner supported the head and neck in the neutral position over the edge of the examination table. Neck rotation ranged from $100^{\circ}$ to $120^{\circ}$ and lateral flexion from $65^{\circ}$ to $75^{\circ}$ in infants aged 2 to 10 months. Cheng et al ${ }^{22}$ assessed the cervical rotation on 821 consecutive patients with congenital muscular torticollis, classified as palpable sternomastoid tumor, muscular torticollis, and postural torticollis. The reproducibility of rotation measurements had an intertester reliability correlation coefficient of 0.71. Likewise, Klackenberg et al examined the lateral flexion in 23 infants with CMT by a goniometer for rotation and a protractor for lateral flexion. This method was found to have high intratester reliability with the interclass correlation coefficient reported as 0.94 to 0.98 . $^{24}$

The main limitation of the present study was the absence of a control group. Moreover, we did not perform a goniometric assessment of cervical ROMs to compare our clinical evaluation with an instrumental gold standard. Finally, follow-up examinations could be interesting to understand how these patients evolve over time.

Infants with PP are likely to have cervical ROM limitations, even in the absence of any structural neck musculature damage. From this point of view, assessing active and passive cervical ROM should be a part of the routine physical examination in patients with PP. Cervical ROM limitation may be detected by means of easy and reliable maneuvers, such as those described in the present paper.

\section{REFERENCES}

1. Collett BR, Gray KE, Starr JR, et al. Development at age 36 months in children with deformational plagiocephaly. Pediatrics 2013;131:e109e115

2. Steinbok P, Lam D, Singh S, et al. Long-term outcome of infants with positional occipital plagiocephaly. Childs Nerv Syst 2007;23:12751283

3. Ottenbacher K. Impact of random assignment on study outcome: an empirical examination. Control Clin Trials 1992;13:50-61

4. Mawji A, Vollman AR, Hatfield J, et al. The incidence of positional plagiocephaly: a cohort study. Pediatrics 2013;132:298-304

5. van Vlimmeren LA, van der Graaf Y, Boere-Boonekamp MM, et al. Effect of pediatric physical therapy on deformational plagiocephaly in children with positional preference: a randomized controlled trial. Arch Pediatr Adolesc Med 2008;162:712-718

6. McKinney CM, Cunningham ML, Holt VL, et al. Characteristics of 2733 cases diagnosed with deformational plagiocephaly and changes in risk factors over time. Cleft Palate Craniofac J 2008;45:208-216

7. Littlefield TR, Beals SP, Manwaring KH, et al. Treatment of craniofacial asymmetry with dynamic orthotic cranioplasty. J Craniofac Surg 1998;9:11-17

8. Mulliken JB, Vander Woude DL, Hansen M, et al. Analysis of posterior plagiocephaly: deformational versus synostotic. Plast Reconstr Surg 1999;103:371-380

9. Bialocerkowski AE, Vladusic SL, Wei Ng C. Prevalence, risk factors, and natural history of positional plagiocephaly: a systematic review. Dev Med Child Neurol 2008;50:577-586

10. Nilesh K, Mukherji S. Congenital muscular torticollis. Ann Maxillofac Surg 2013;3:198-200

11. Pivar SJ, Scheuerle A. Variable co-diagnosis of plagiocephaly and torticollis in Texas health care facilities. J Craniofac Surg 2006;17: 236-240

12. Hutchison BL, Thompson JM, Mitchell EA. Determinants of nonsynostotic plagiocephaly: a case-control study. Pediatrics 2003;112:e316

13. Karski J, Karski T, Kendzierski G, et al. "Contracture syndrome" in newborns and infants according to Prof. Hans Mau as an explanation of the "geography" and certain clinical features of idiopathic scoliosis. Ortop Traumatol Rehabil 2005;7:23-27 
14. Argenta L, David L, Thompson J. Clinical classification of positional plagiocephaly. J Craniofac Surg 2004;15:368-372

15. Spermon J, Spermon-Marijnen R, Scholten-Peeters W. Clinical classification of deformational plagiocephaly according to Argenta: a reliability study. J Craniofac Surg 2008;19: 664-668

16. Stellwagen L, Hubbard E, Chambers C, et al. Torticollis, facial asymmetry and plagiocephaly in normal newborns. Arch Dis Child 2008;93:827-831

17. Hanney WJ, George SZ, Kolber MJ, et al. Inter-rater reliability of select physical examination procedures in patients with neck pain. Physiother Theory Pract 2014;30:345-352

18. Fowler J, Jarvis P, Chevannes M. Practical Statistics for Nursing and Health Care. West Sussex, UK: Wiley; 2002:213 p

19. Cabrera-Martos I, Valenza MC, Valenza-Demet G, et al. Impact of torticollis associated with plagiocephaly on infants' motor development. J Craniofac Surg 2015;26:151-156
20. Rogers GF, Oh AK, Mulliken JB. The role of congenital muscular torticollis in the development of deformational plagiocephaly. Plast Reconstr Surg 2009;123:643-652

21. Williams MA, McCarthy CJ, Chorti A, et al. A systematic review of reliability and validity studies of methods for measuring active and passive cervical range of motion. $J$ Manipulative Physiol Ther 2010;33:138-155

22. Cheng JCY, Wong MWN, Tang SP, et al. Clinical determinants of the outcome of manual stretching in the treatment of congenital muscular torticollis in infants. A prospective study of eight hundred and twentyone cases. J Bone Joint Surg Am 2001;83-A:679-687

23. Christensen C, Landsettle A, Antoszewski S, et al. Conservative management of congenital muscular torticollis: an evidence-based algorithm and preliminary treatment parameter recommendations. Phys Occup Ther Pediatr 2013;33:453-466

24. Ohman AM, Beckung ER. Reference values for range of motion and muscle function of the neck in infants. Pediatr Phys Ther 2008;20:53-58

\section{The Effect of Cigarette Smoking on the Healing of Extraction Sockets: An Immunohistochemical Study: ERRATUM}

In the article that appeared on page e397 of the July 2014 issue of the Journal of Craniofacial Surgery, an author's name did not appear. The full list of authors and affiliations is as follows:

Aydin Ozkan, DDS, PhD, ${ }^{*}$ Gurkan Rasit Bayar, DDS, PhD, ${ }^{\dagger}$ Hasan Ayberk Altug, DDS, PhD, ${ }^{\dagger}$ Metin Sencimen, DDS, PhD, ${ }^{\dagger}$ Necdet Dogan, DDS, PhD, ${ }^{\dagger}$ Yilmaz Gunaydin, DDS, PhD, ${ }^{\dagger}$ and Esra Erdogan, $\mathrm{MD}^{\ddagger}$

From the Departments of ${ }^{*}$ Oral \& Maxillofacial Surgery, Diyarbakir Military Hospital, Diyarbakir; ${ }^{\dagger}$ Oral \& Maxillofacial Surgery and ${ }^{\ddagger}$ Histology and Embryology, Gulhane Military Medical Academy, Ankara, Turkey.

\section{REFERENCES}

Ozkan A, Bayar GR, Altug HA, et al. The effect of cigarette smoking on the healing of extraction sockets: an immunohistochemical study. J Craniofac Surg 25;4:e397-e402

Ozkan A, Bayar GR, Altug HA, et al. The effect of cigarette smoking on the healing of extraction sockets: an immunohistochemical study: erratum. J Craniofac Surg 25;6:2273 